\title{
Schott will bis 2030 klimaneutral werden
}

\author{
Der Spezialglaskonzern stellt sich den Herausforderungen des Klimawandels und plant, sich \\ bis 2030 zu einem klimaneutralen Unternehmen weiterzuentwickeln, um damit einen aktiven \\ Beitrag für den Klimaschutz zu leisten. Das Projekt ,Zero Carbon' ist Bestandteil der neuen \\ Konzernstrategie.
}

Mit dem Strategieprojekt ,Zero Carbon` beginnt Schott eine neue Ära seines Umwelt- und Klimamanagements. Als Spezialglashersteller gehört das Unternehmen einer energieintensiven Industriebranche an. Spezialgläser und Glaskeramiken werden in großen Schmelzwannen bei Temperaturen von bis zu $1.700{ }^{\circ} \mathrm{C}$ geschmolzen. Bisher werden die Schmelzwannen mit den fossilen Energieträgern Erdgas und Heizöl oder mit Elektrizität beheizt. Auch bei der Weiterverarbeitung der Gläser wird viel Energie benötigt. Durch diesen Energiebedarf beträgt der klimarelevante Fußabdruck rund eine Million Tonnen $\mathrm{CO}_{2} \mathrm{e}$ ( $\mathrm{CO}_{2}$-Äquivalente) pro Jahr.

„In unserem Engagement für den Klimaschutz sehen wir drei Schritte: Zunächst wollen wir möglichst viele klimaschädliche Emissionen vermeiden, nicht vermeidbare Emissionen im nächsten Schritt deutlich reduzieren und, falls nicht anders möglich, schließlich die restlichen Emissionen kompensieren“, sagte Frank Heinricht, Vorsitzender des Vorstandes. Damit leistet das Unternehmen einen Beitrag zu den Zielsetzungen des Pariser Klimaschutzabkommens.

Der Aktionsplan zur Klimaneutralität umfasst vier Handlungsfelder:

- Verbesserung der Energieeffizienz

- Umstieg auf Grünstrom

- Technologiewandel

- Kompensation technologisch nicht vermeidbarer Emissionen

Die Verbesserung der Energieeffizienz hat das Unternehmen schon seit Jahrzehnten im Blick. Durch die Einführung der Oxy-FuelSchmelztechnologie und die zunehmende Nutzung von Strom zur Beheizung der Schmelzwannen seit den 1990er Jahren ist es bereits gelungen, den spezifischen Energieverbrauch um über $30 \%$ zu senken. Im Rahmen eines Energiemanagementsystems arbeiten
Experten intensiv daran, weitere Einsparpotenziale zu identifizieren und zu nutzen.

\section{Umstieg auf Grünstrom bis 2021}

Bei elektrischer Energie setzt Schott künftig vollständig auf Grünstrom. Bereits 2021 will der Konzern seinen Strombedarf zu 100 \% durch erneuerbare Energien wie Wasserkraft, Windkraft, Solarenergie und Biomasse decken. Hierbei wird Wert darauf gelegt, dass der Grünstrom eine hochwertige Zertifizierung aufweist und so einen starken Beitrag zur Energiewende leistet.

\section{Neue Technologien mit Fokus auf Wasserstoff}

Langfristig soll ganz auf die Nutzung fossiler Energieträger verzichtet werden, soweit es technologisch machbar ist. „Dieser Transformationsprozess braucht allerdings Zeit und erfordert hohe Entwicklungs- und Investitionskosten“, so Heinricht. Einen vielversprechenden Lösungsansatz sieht das Unternehmen dabei in der Wasserstofftechnologie. Daneben prüfen Forscher und Schmelztechnologen die Machbarkeit weiterer technologischer Ansätze. „Wir glauben, dass wir als Innovationstreiber unserer Branche in den nächsten Jahren und Jahrzehnten hier wichtige Impulse setzen können“, sagte Frank Heinricht.

\section{Kompensation technologisch unvermeidbarer Emissionen}

Bis $\mathrm{CO}_{2}$-freie Lösungen für die Beheizung großer Glasschmelzwannen zur Verfügung stehen, will Schott technologisch nicht vermeidbare Emissionen durch Investitionen in Klimaschutzprojekte kompensieren.

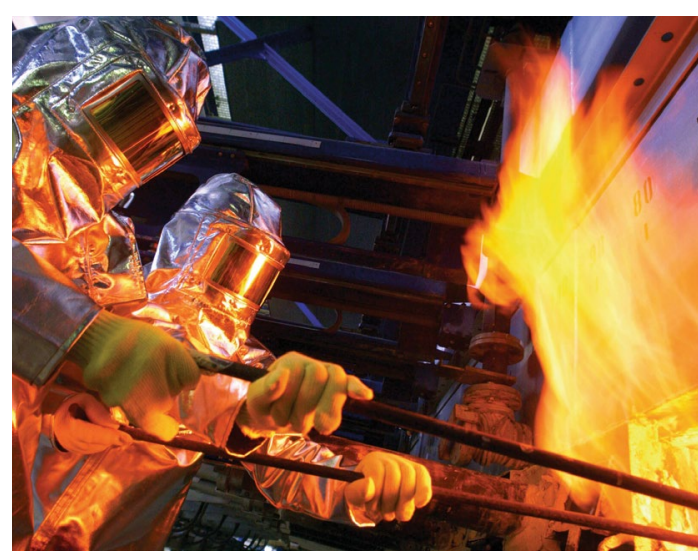

Die Herstellung von Spezialgläsern ist energieintensiv: Sie werden bei Temperaturen von bis zu $1.700{ }^{\circ} \mathrm{C}$ geschmolzen. (๑ Schott)

Derzeit erstellt der Konzern ein Kompensationsportfolio, das hohe Anforderungen an die Nachhaltigkeit der Projekte erfüllt.

Zur Berechnung seiner klimarelevanten Emissionen betrachtet Schott den Ausstoß aller Treibhausgase. Dabei werden aktuell die Emissionen aus der eigenen Produktion (Scope 1 des Greenhouse Gas Protocol) und aus eingekaufter Energie (Scope 2) in die Berechnung einbezogen. Von Scope 3 werden auch die Dienstreisen und die Mobilität der Mitarbeiter berücksichtigt. Mittelfristig sollen dann die weiteren Scope3-Emissionen mit einbezogen werden, die unter anderem auch die Emissionen in der Lieferkette betrachten. Um eine Vergleichbarkeit bezüglich ihrer Klimawirksamkeit herzustellen, werden die Treibhausgasemissionen in $\mathrm{CO}_{2}$ - Äquivalenten $\left(\mathrm{CO}_{2} \mathrm{e}\right)$ angegeben.

Kontakt:

Schott AG, Mainz, www.schott.com/german 